\title{
Degradation and Viscoelastic properties of PLA-PCL, PGA-PCL, PDO and PGA fibres
}

\author{
A.C. Vieira ${ }^{1,2, a}$, J.C. Vieira ${ }^{1, b}$, R.M. Guedes ${ }^{2, ~ c}$, A.T. Marques ${ }^{2, d}$ \\ ${ }^{1}$ Unidade de Materiais e Estruturas Compósitas, INEGI, \\ Rua Roberto Frias 400, 4200-465 Porto, Portugal \\ ${ }^{2}$ Departamento de Engenharia Mecânica e Gestão Industrial (DEMEGI), \\ Faculdade de Engenharia, Universidade do Porto (FEUP), \\ Rua Roberto Frias s/n, 4200-465 Porto, Portugal

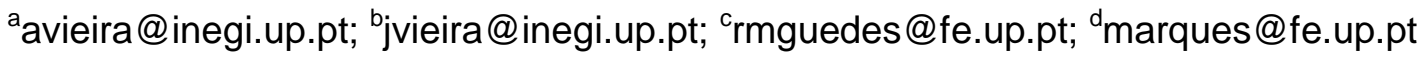

Keywords: mechanical properties, degradation rate, strain rate, aliphatic polyesters

\begin{abstract}
Aliphatic polyesters, such as polylactic acid (PLA), polyglycolic acid (PGA), polycaprolactone (PCL), polydioxone (PDO) and others, have been commonly used in biodegradable products. Hydrolytic and/or enzymatic chain cleavage of these materials leads to $\alpha$ hydroxyacids, which, in most cases, are ultimately assimilated in human body or in a composting environment. However, each of these has some shortcomings, in terms of mechanical properties and degradation time, which restrict its applications. The combination of these materials, by copolymerization or blending, enables a range of mechanical properties and degradation rates. These are extremely promising approaches which can improve or tune the original properties of the polymers. A composite solution of several materials with different degradation rates also enables tuning the rate of degradation of a device and the mechanical properties.

After immersion of an aliphatic polyester device, diffusion occurs very rapidly compared to hydrolysis. Therefore, it is usually considered that hydrolysis of ester bonds starts homogeneously and has traditionally been modelled according to a first order kinetics.

In this experimental study, fibres of PLA-PCL, PGA-PCL, PDO and PGA, with two different dimensions, were characterized in terms of their degradation rate under three different environments (water, $\mathrm{NaCl}$ and $\mathrm{PBS}$ ) at constant temperature $\left(37^{\circ} \mathrm{C}\right)$. Weights and mechanical properties were measured after six different degradation stages. Stages durations were different depending on materials, according to the predicted degradation times. As other thermoplastics, they are viscoelastic materials. In this experimental study mechanical properties of fibres were compared at different strain rates.
\end{abstract}

\section{Introduction}

Biodegradable polymers have been used in a variety of commodity devices and biomedical applications. In biomedical application, their main application is on regenerative medicine, where they temporarily replace the biomechanical functions while the natural tissue regenerates; or in controlled drug delivery, as reviewed by Nair L. and Laurencin C. [1]. Hence a surgery to remove the device is not required, since it will be naturally metabolized. In the case of commodity products, biodegradable plastics claim clear environmental advantages in several brief use applications, mainly in their final stage of life (waste disposal), which can clearly be evident through life cycle assessment. 
Performance of a device depends of its behaviour to a mechanical, thermal or chemical applied stress. It is mostly conditioned by the materials selection and dimensioning of the product. For a biodegradable product, performance will decrease along its degradation. From the final user point of view, performance should be enough for the predicted use, during all its life cycle. Biodegradable plastics can present short term performances similar to conventional plastics. There are many biodegradable polymers commercially available to produce a great variety of plastic products, each of them with suitable properties according to the application. So, it is possible to find a large range of mechanical properties and degradation rates useful for a great variety of products for brief use.

The poly- $\alpha$-hydroxyesters, polylactic acid (PLA), polyglycolic acid (PGA) and copolymers are the most popular aliphatic polyesters that have been synthesized for more than 30 years. The lefthanded (L- lactide) and right-handed (D-lactide) are the two enantiometric forms of PLA, with PDLA having a much higher degradation rate than PLLA. An intensive overview was done by Auras R. Et al. [2]. PLLA is a rather brittle polymer with a low degradation rate, and compounding with PCL is frequently employed to improve mechanical properties. PCL is also hydrophilic with a low degradation rate, much more ductile than PLA [3]. PGA, since it is a hydrophilic material presents a high degradation rate. The combination of PGA with PLA is usually employed to tune degradation rate [1]. Polyhydoxyalkanoates (PHA's) is the largest class of aliphatic polyesters, comprising poly 3-hydroxybutyrate (PHB), copolymers of 3-hydroxybutyrate and 3hydroxyvalerate (PHBV), poly 4-hydroxybutyrate (P4HB), copolymers of 3-hydroxybutyrate and 3-hydroxyhexanoate (PHBHHx) and poly 3-hydroxyoctanoate (PHO) and its blends. The changing PHA compositions also allow favourable mechanical properties and degradation times within desirable time frames [4]. They represent a wide range of mechanical properties and degradation times, as can be seen in Fig. 1.

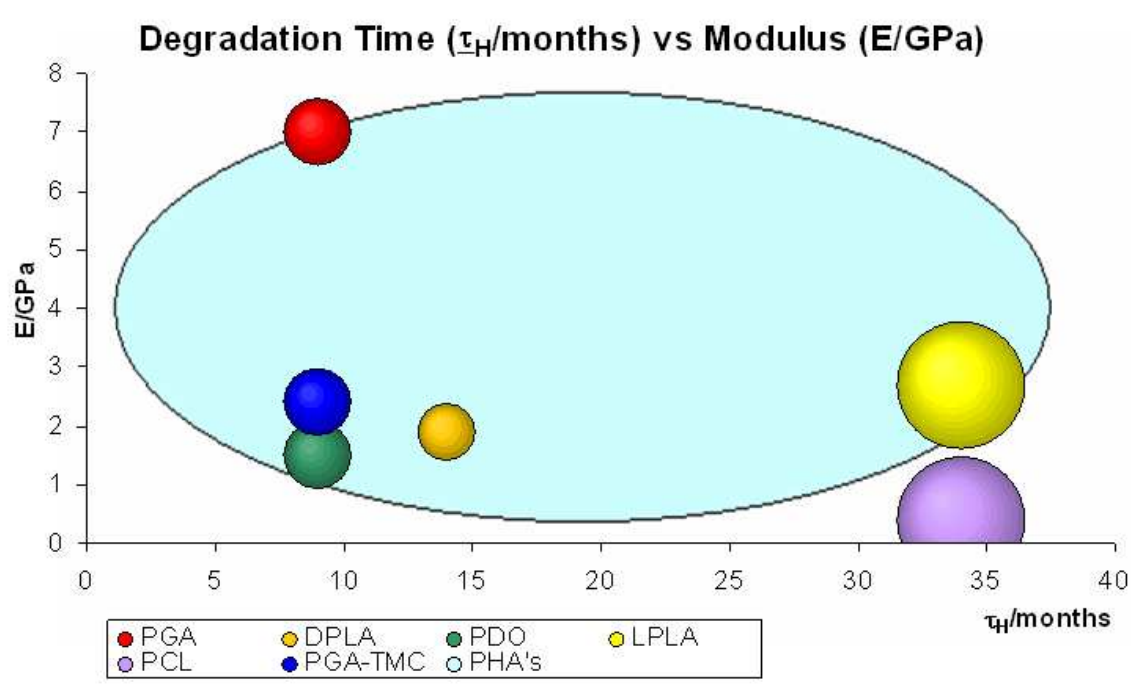

Figure 1 - Range of mechanical properties and mass loss degradation times

Biodegradable plastics, as other thermoplastics, are viscoelastic materials. This means that mechanical properties, like stiffness, are time dependent and, therefore, stress/strain rate dependent. For instance, at high strain rates they present a more brittle behaviour, with a transition from ductile to brittle behaviour above a specific strain rate. A general consequence of hydrolytic degradation is the lowering of the plastic flow ability of the polymer, thus causing the change from ductile behaviour into a brittle one or, if the behaviour was initially brittle, an increase in the brittleness. After hydrolysis has started, brittleness will occur at lower strain rates. On the other hand it is 
expected that transition strain rate will increase with swelling. After water absorption, a biodegradable plastic presents a stiffness decrease.

\section{Hydrolytic degradation}

After immersion of an aliphatic polyesters device in an aqueous medium, the very first event which occurs is water uptake. The penetrating water rapidly creates a negative gradient of water concentrations from the surface to the centre as expected from a pure diffusion viewpoint. However, this gradient vanishes in a couple of hours or days, depending on temperature, thickness or material hydrophobicity. Diffusion of small molecules, like water, is much fast compared with degradation. Therefore, one can consider that hydrolysis of ester bonds starts homogeneously.

For aliphatic polyesters, hydrolysis rates are affected by the temperature, molecular structure, ester group density and by the degradation medium. The degree of crystallinity may be a crucial factor, since reaction medium attack mainly the amorphous domains of a polymer [5]

Hydrolysis has traditionally been modelled using a first order kinetics based on the kinetic mechanism of hydrolysis, according to the Michaelis-Menten scheme [6]. According to Farrar and Gillson [7] the following first-order equation describes the hydrolytic process to occur relative to the carboxyl end groups (C), ester concentration (E) and water concentration (W):

$$
\frac{d C}{d t}=-k E W C=-u_{m} C
$$

where $u_{m}$ is the medium hydrolysis rate of the material, $E$ and $W$ are constant in the early stages of the reaction. In addition, water is spread out uniformly in the sample volume (no diffusion control). Using the molecular weight, and since the concentrations of carboxyl end groups $C=1 / M_{n_{t}}$; the equation 1 will become:

$$
M_{n_{t}}=M_{n_{0}} e^{-u_{m} t}
$$

where $M_{n_{t}}$ and $M_{n_{0}}$, are the number-average molecular weight, at a given time $t$ and initially at $t=0$, respectively.

In the design phase of a biodegradable device, it is important to predict the evolution of strength, instead of molecular weight. It has been shown [8] that the tensile strength of a polymer can, in many cases, be related to $M_{n}$ through the relationship:

$$
\sigma=\sigma_{\infty}-\frac{B}{M_{n_{t}}}=\sigma_{\infty}-\frac{B}{M_{n_{0}} e^{-k W t}}
$$

where $\sigma$ is the fracture strength, $\sigma_{\infty}$ is the fracture strength at infinite molecular weight, and $B$ is an empirical constant of the material. One can thus determine the limit strength for the material that can weaken during time, $\sigma_{d}=f(t)$.

\section{Experimental Procedure}

Four different biodegradable thermoplastics (PGA, PLA-PCL, PGA-PCL and PDO), representing a wide range of degradation times and mechanical properties, were provided by Chirmax. Suture fibres with two different diameters $(150 \mu \mathrm{m}$ and $400 \mu \mathrm{m})$ were selected for measuring degradation 
properties. Three test pieces of $100 \mathrm{~mm}$ long each were cut from sterile suture fibres in biological safety chamber to avoid contamination. Fibres specimens were then placed in $50 \mathrm{ml}$ test tubes and submitted to six different degradation stages under water, saline solution and PBS (phosphate buffer solution), at constant temperature $\left(37^{\circ} \mathrm{C}\right)$ in a thermostatic bath. The duration of stages was previously determined, according to the supplier durability claims, until a maximum of 7 months.

Dry weight $\left(24 \mathrm{~h}\right.$ in incubator at $\left.37^{\circ} \mathrm{C}\right)$ was measured initially and at the end of each stage, to determine material erosion. Number-average molecular weight was determined at the end of each stage, by GPC (Gel Permeation Chromatography), for PLA-PCL specimens, using chloroform as solvent and eluent. Mechanical properties were also repeatedly evaluated at the end of each stage. A universal mechanical test machine was used, with a load cell off $100 \mathrm{~N}$, and pneumatic grips commonly used in fibre testing. The distance between grips was set for $50 \mathrm{~mm}$. Three specimens were used for each fibre sample. The displacement rate was $500 \mathrm{~mm} / \mathrm{min}$. The evolution curve of these physical properties was determined during degradation using this methodology.

In addition, mechanical properties of undegraded PLA-PCL fibres, with diameter of $400 \mu \mathrm{m}$, were similarly tested at different displacement rates $(15 \mathrm{~mm} / \mathrm{min}, 30 \mathrm{~mm} / \mathrm{min}, 60 \mathrm{~mm} / \mathrm{min}, 125 \mathrm{~mm} / \mathrm{min}$ and $250 \mathrm{~mm} / \mathrm{min}$ ). Another saturated sample was tested at $500 \mathrm{~mm} / \mathrm{min}$.

\section{Results}

In Fig. 2 experimental results of weight loss are presented for the four materials immersed in the three degradation media.

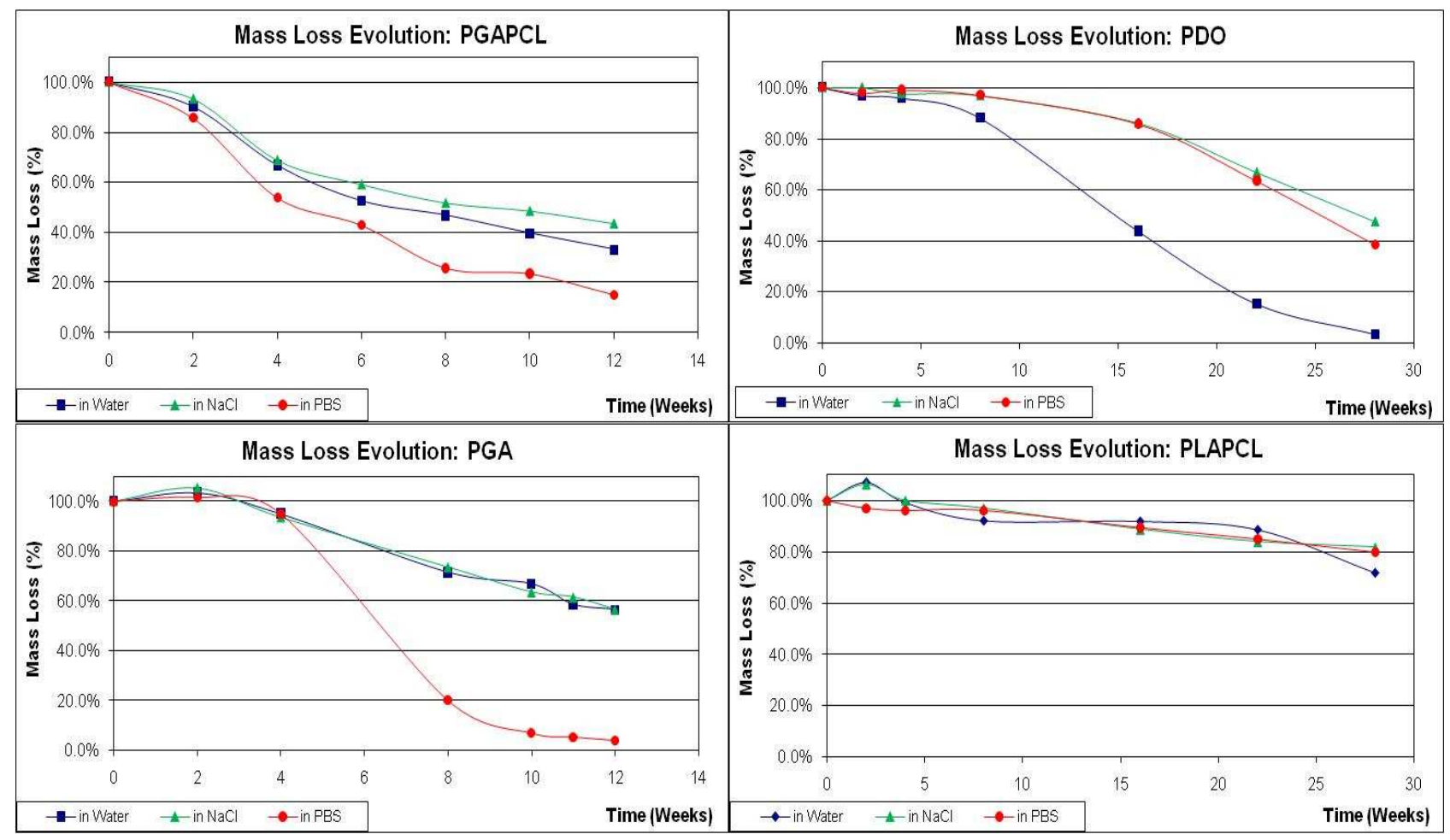

Figure 2 - Normalised weight loss of PGA-PCL, PDO, PGA and PLA-PCL suture fibres

PGA-PCL fibres are the fastest in terms of weight loss. Although PCL has a low degradation rate, the combination with PGA results in a less crystalline plastic, and this may explain the faster degradation, as it occurs with PLGA [1]. It has become very fragile just after 2 weeks. It is followed by PGA, PDO and the less degradable PLA-PCL. Alkaline solution of PBS is the fast degrading 
media. Except for PDO, where water with $\mathrm{pH}$ closed to neutral, degrades much faster than the other two media. For PLA-PCL, no significant differences were observed between the three media.

After two weeks of degradation, maximum load of PGA decreased from $15 \mathrm{~N}$ to around 2,5 $\mathrm{N}$ for the small diameter fibres and from $80 \mathrm{~N}$ to about $15 \mathrm{~N}$ for the larger diameter fibres. Their rupture has become brittle, not showing any plastic deformation. There were no significant differences between dimensions, meaning that diffusion has neglective influence on degradation.

Comparing the normalised strength evolution of PDO suture fibres, significant differences between dimension were observed. These were more significant for slower degrading media $(\mathrm{NaCl}$ and PBS). A faster degradation is observerved for the biger diameter fibres, probably due to a more cristalline structure of small fibers. Water was much faster to degrade PDO strength, while no significant differences in degradation rate was found between the other two media.

PLA-PCL has become brittle only after 16 weeks, but strength was progressively reduced (see Fig.3). No significant differences between dimensions were observed. One can assume that, also for PLA-PCL, diffusion has neglective influence on degradation and hydrolysis starts homogeneously.

No tensile test results were available for PGA-PCL, since it was already very fragile without degradation, and it was impossible to test it after two weeks of degradation.
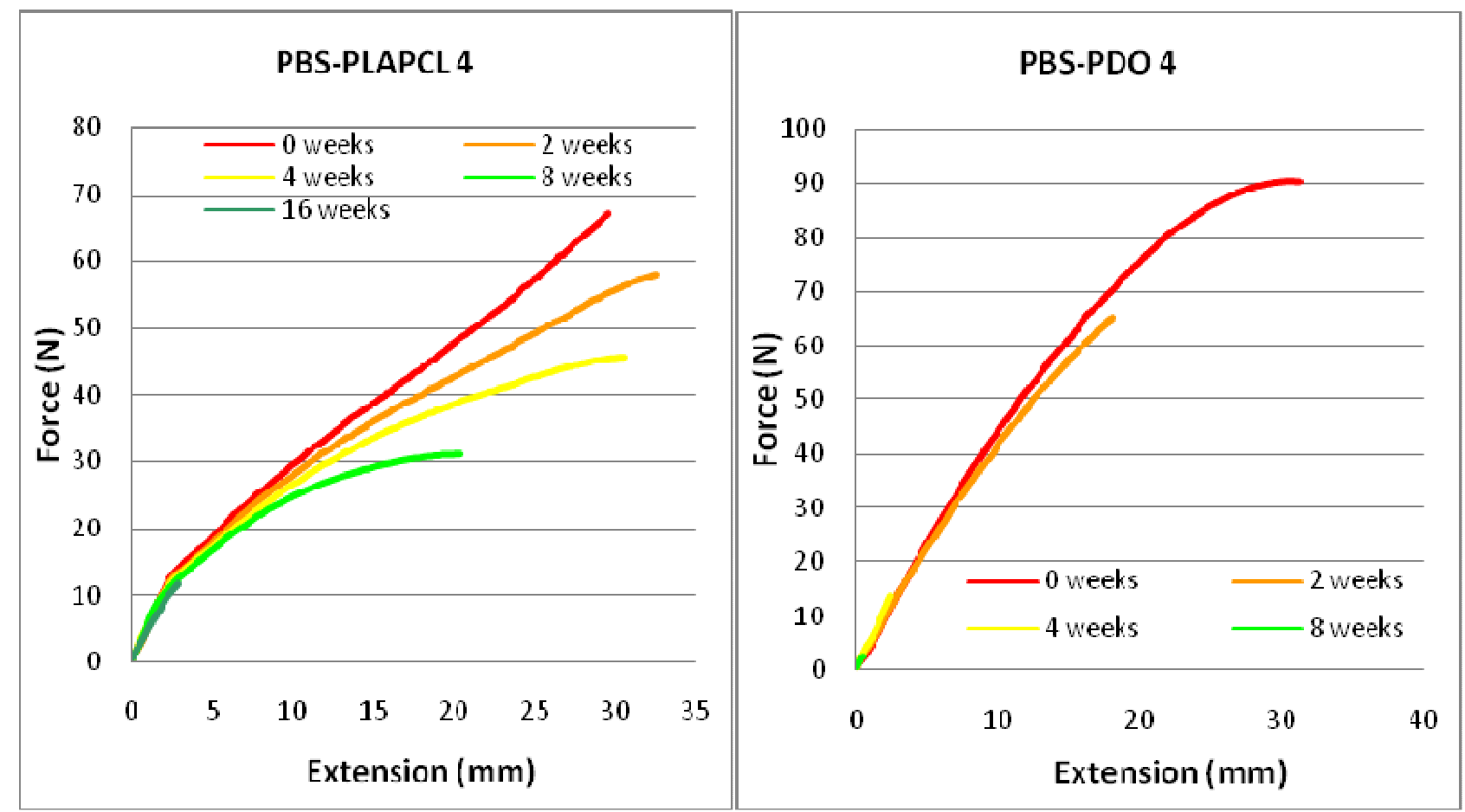

Figure 3 - Tensile test results along PLAPCL (left) and PDO (rigth) degradation in phosphate buffer solution (PBS), of suture fibres UP4 $(400 \mu \mathrm{m})$

From Fig. 3, no significant variation of Young modulus along degradation was found for the materials studied in this experimental work. In table 2, the degradation rate of strength is presented, for the three materials and the three medium. The degradation rate of strength for PDO incubated in water is about twice of the other media. Comparing to tensile results of Fu et. al [9] of PLGA with a degradation rate of strength, $k$, of around 0.9 is slightly higher than that of PGA alone. Once again, this is due to a less crystalline structure. In Fig. 4, relative molecular weight and relative strength are presented in logarithmic scale. These have a proportional evolution, in agreement to Ward [8]. 
Table 2 - Degradation rate of strength of PLA-PCL, PDO and PGA incubated in water, saline and $\mathrm{PBS}$ at $37^{\circ} \mathrm{C}$

\begin{tabular}{|c|c|c|}
\hline \multicolumn{2}{|c|}{$\operatorname{In}\left(\boldsymbol{\sigma} / \boldsymbol{\sigma}_{\mathrm{o}}\right)=-\mathbf{k t}$} & $\mathbf{k}$ \\
\hline \multirow{3}{*}{ PLAPCL } & water & 0,092 \\
\cline { 2 - 3 } & $\mathrm{NaCl}$ & 0,095 \\
\cline { 2 - 3 } & PBS & 0,103 \\
\hline \multirow{3}{*}{ PDO } & water & 1,481 \\
\cline { 2 - 3 } & $\mathrm{NaCl}$ & 0,475 \\
\cline { 2 - 3 } & PBS & 0,483 \\
\hline \multirow{3}{*}{ PGA } & water & 0,848 \\
\cline { 2 - 3 } & $\mathrm{NaCl}$ & 0,752 \\
\cline { 2 - 3 } & PBS & 0,797 \\
\hline
\end{tabular}

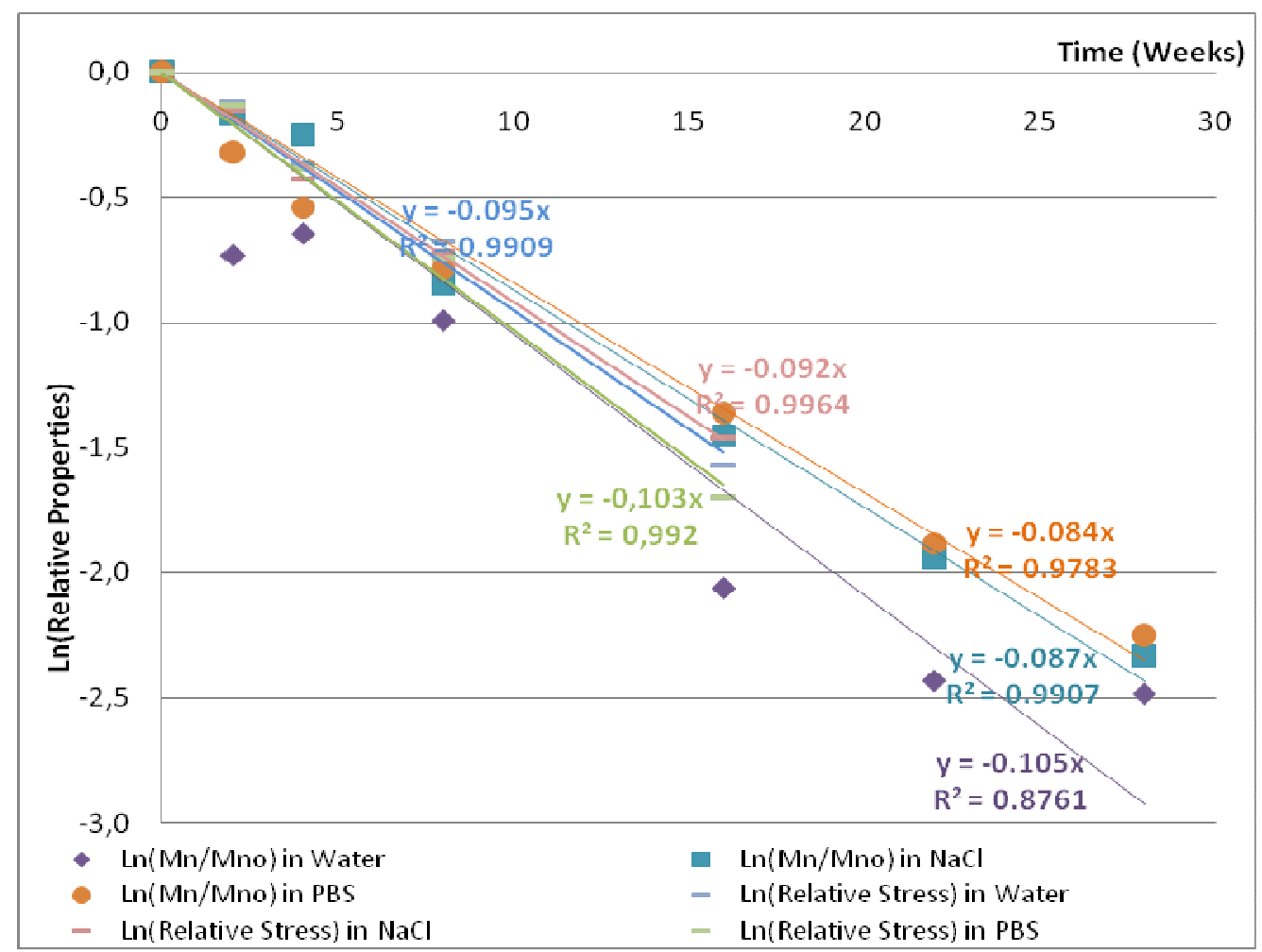

Figure 4 - Normalised molecular weigth $\left(\mathrm{Mn} / \mathrm{Mn}_{\mathrm{o}}\right)$ and normalised tensile strength $\left(\sigma / \sigma_{\mathrm{o}}\right)$ evolutions, for PLA-PCL suture fibres of UP4 $(400 \mu \mathrm{m})$, incubated in water, saline solution and PBS

According to the predicted behaviour, Young modulus and yield strength of PLA-PCL increases with increasing strain rate (see Fig. 5). This phenomenon is expected to be more significant with more hydrophilic materials like PGA. 


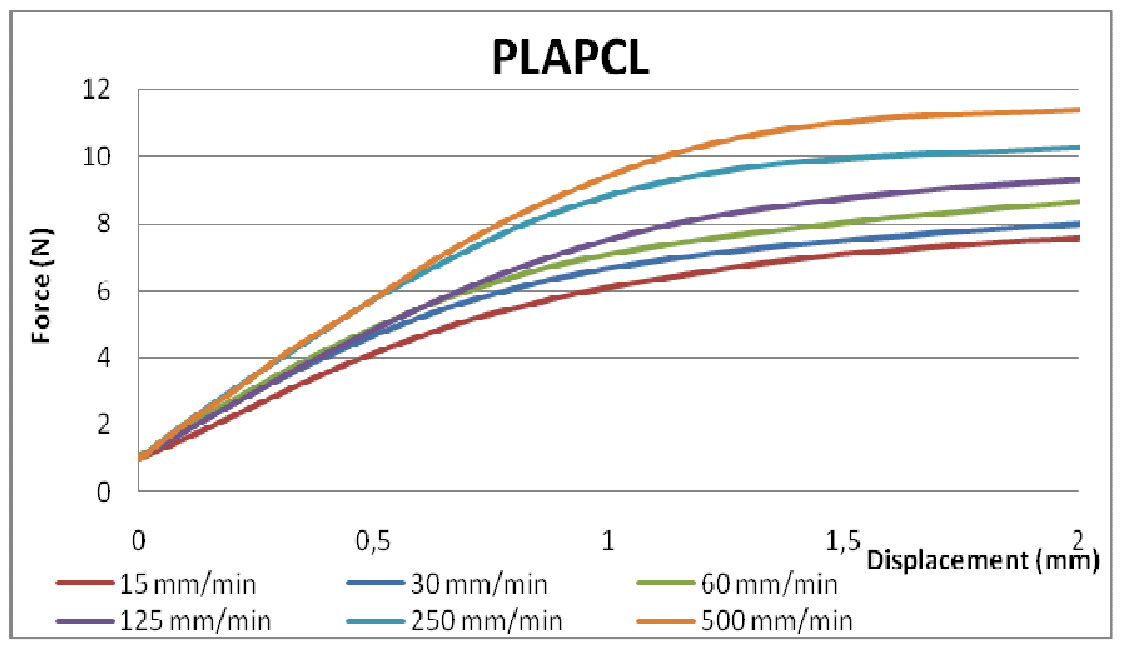

Figure 5 - Tensile test curves of PLA-PCL at diferent displacement rates

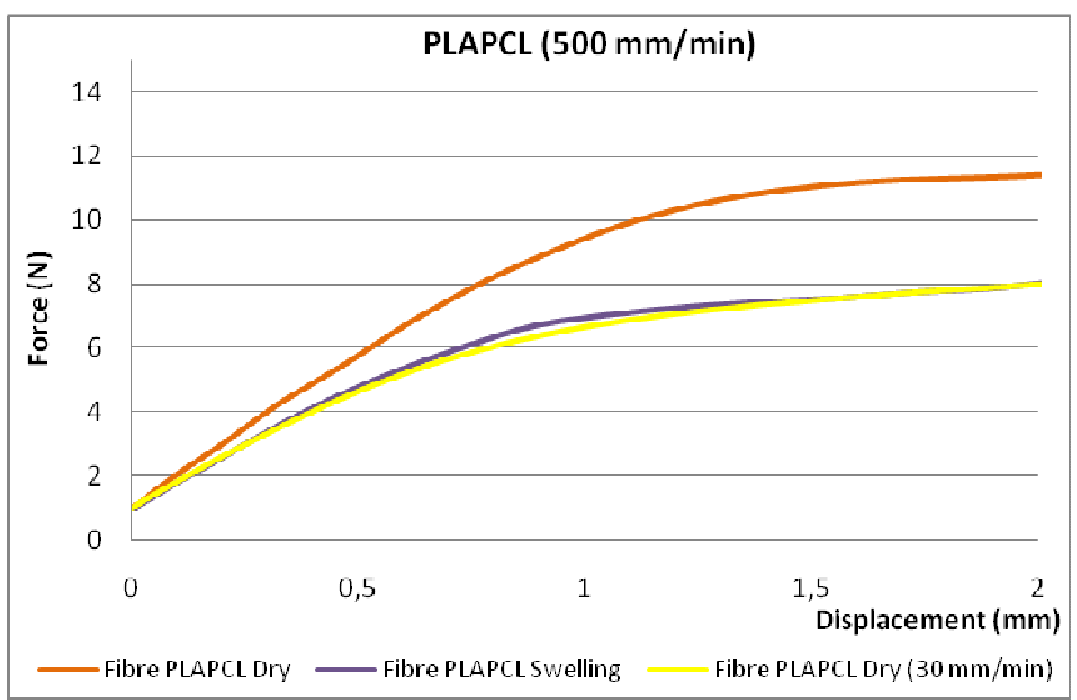

Figure 6 - Tensile test curves of dry and satureted PLA-PCL, loaded at 500 $\mathrm{mm} / \mathrm{min}$, and dry, loaded at $30 \mathrm{~mm} / \mathrm{min}$

After swelling, the PLA-PCL fibre loaded at $500 \mathrm{~mm} / \mathrm{min}$ presents a stiffness decrease. In this case Young modulus and yield strength were similar when the dry fibre was loaded at $30 \mathrm{~mm} / \mathrm{min}$ (see figure 6).

\section{Conclusions}

Mechanical properties and degradation rates can be tailored to match the device requirements. Tensile strength evolution can be determined along degradation time in test specimens. This is possible since hydrolytic reaction is the limiting step of overall degradation process. Diffusion may be neglected in most cases, particularly for small dimensions, and hydrolysis may be considered to start homogeneously along the volume. The degradation rate can be further used in the dimensioning of biodegradable devices, since tensile strength depends on molecular weight. However, stiffness is independent of molecular weight.

Mechanical properties of aliphatic polyesters will depend on the displacement rate applied and water uptake. These must be determined bearing in mind the application. 


\section{Acknowledgments}

The authors would like to thank FCT for financial support under the grant PTDC/EMEPME/70155/2006, and CHIRMAX for material supply.

\section{References}

[1] Nair, L.S.; Laurencin, C.T., Biodegradable polymers as biomaterials. Prog. Polym. Sci., Vol.32 (2007), p. 762-98.

[2] Auras, R.; Harte, B.; Selke, S., An Overview of Polylactides as Packaging Materials. Macromol. Biosci., Vol. 4 (2004), p. 835-64.

[3] Södergard, A.; Stolt, M., Properties of lactic acid based polymers and their correlation with composition, Prog. Polym. Sci., Vol. 27 (2002), p. 1123-63.

[4] Chen, G.-Q.; Wu, Q., Review:The application of polyhydroxyalkanoates as tissue engineering materials. Biomaterials, Vol. 26 (2005), p. 6565-78.

[5] Marten, E.; Muller, R.J.; Deckwer, W.D., Studies on the enzymatic hydrolysis of polyesters - II. Aliphaticearomatic copolyesters. Polymer Degradation and Stability, Vol. 88 (2005), p. 371-81.

[6] Bellenger, V.; Ganem, M.; Mortaigne, B.; Verdu, J., Lifetime prediction in the hydrolytic ageing of polyesters. Polymer Degradation and Stability, Vol, 49 (1995), p. 91-7.

[7] Farrar, D.F.; Gillson, R.K., Hydrolytic degradation of polyglyconate B: the relationship between degradation time,strength and molecular weight. Biomaterials, Vol. 23 (2002), p. 3905-12.

[8] Ward, I.M., in: Mechanical properties of solid polymers. 2nd ed., edit by John Wiley \& Sons, Chichester, UK (1983).

[9] Fu, B.X.; Hsiao, B. S.; Chen, G.; Zhou, J.; Koyfman, I.; Jamiolkowski, D.D.; Dormier, E., Structure and property studies of bioabsorbable poly(glycolide-co-lactide) fiber during processing and in vitro degradation. Polymer, Vol.43 (2002), p. 5527-34. 